\title{
Neuer, elektronischer Meldeweg für die Todesursachen
}

Dr. med. Monika Diebold, Dr. med. Christoph Junker

Bundesamt für Statistik, Sektion Gesundheit
In der Schweiz werden die Todesursachen seit 1874 erfasst und statistisch aufbereitet. Es ist klar, dass sich über die Jahre vieles dabei geändert hat. Jetzt steht ein neuer Entwicklungsschritt an: Ab dem 1. Mai 2006 wird es den Ärztinnen und Ärzten möglich sein, die Todesursachen auf elektronischem Weg an das Bundesamt für Statistik (BFS) zu übermitteln.

Das BFS unternimmt diesen Schritt nicht nur, um mit der technischen Entwicklung Schritt zu halten, sondern auch aus ganz praktischen Gründen. Pro Jahr erhalten wir über 60000 Meldungen, zumeist handschriftlich, die alle entziffert und anschliessend codiert werden müssen. Bei vielen wäre eine automatisierte Weiterverarbeitung möglich, doch dazu müssten die Angaben in maschinenlesbarer Form vorliegen. Auch bei der Handverarbeitung ist eine gut lesbare Maschinenschrift von grossem Vorteil.
Deshalb bitten wir Sie, der elektronischen Meldung wenn immer möglich den Vorrang zu geben. Die Meldung auf dem Papierformular wird aber weiterhin möglich sein.

Inhaltlich ändert sich bei der Bescheinigung der Todesursachen nichts. Das Formular wird weiterhin vom Zivilstandsamt dem Arzt geschickt, welcher den Tod bescheinigt hat. Auf dem Formular ist das Vorgehen für die elektronische Meldung beschrieben. Das BFS hat versucht, diesen möglichst einfach zu gestalten. Der Datenschutz wird auch mit der neuen Form gewährleistet, die Namen der Verstorbenen werden nicht an das BFS übermittelt. Für den Fall, dass die ärztliche Bescheinigung weiterhin auf Papier erfolgt, wird ein an das BFS adressiertes Antwortcouvert beiliegen.

\section{Communication électronique des causes de décès}

\section{Dr Monika Diebold, Dr Christoph Junker}

Office fédéral de la statistique, section Santé
Les causes de décès sont recensées et exploitées à des fins statistiques depuis 1874 en Suisse. Beaucoup de choses ont bien entendu changé depuis cette date. Et une nouvelle étape sera franchie prochainement: à partir du $1^{\text {er }}$ mai 2006, les médecins auront la possibilité de communiquer les causes de décès par voie électronique à l'Office fédéral de la statistique (OFS).

Cette nouvelle solution leur est proposée pour suivre le progrès technique certes, mais aussi pour des raisons pratiques. Chaque année, l'OFS reçoit 60000 communications de causes de décès, pour la plupart manuscrites, qu'il doit déchiffrer et coder. Pour pouvoir automatiser le traitement de la plupart de ces communications, il faudrait que les indications fournies puissent être lues par les machines, c'est-à-dire qu'elles soient rédigées en caractères d'imprimerie bien lisibles.
S'il est toujours possible d'utiliser le formulaire imprimé, nous vous invitons tout de même à donner la priorité à la voie électronique pour la communication des causes de décès.

Le contenu de l'attestation médicale des causes de décès reste inchangé. Le formulaire correspondant est toujours envoyé par l'office d'état civil au médecin qui a constaté le décès. Ce formulaire comprend entre autres la description de la procédure à suivre pour la communication électronique des causes de décès. L'OFS l'a simplifié le plus possible, tout en veillant à garantir la protection des données; les noms des personnes décédées ne sont pas communiqués à l'OFS. Une enveloppe-réponse adressée à l'OFS sera jointe pour les cas où l'attestation médicale serait effectuée sur papier. 